\title{
Effects of Feed Species and HUFA Composition on Survival and Growth of the Longsnout Seahorse (Hippocampus reidi)
}

\section{OPEN ACCESS}

Edited by:

Simone Libralato,

Istituto Nazionale di Oceanografia e di

Geofisica Sperimentale, Italy

Reviewed by:

Elisabetta Betulla Morello, Institute of Marine Sciences - ISMAR -

Cnr, Italy

Michele Gristina, Consiglio Nazionale delle Ricerche,

Italy

*Correspondence:

Patrick Schubert

patrick.schubert@

allzool.bio.uni-giessen.de

${ }^{\dagger}$ Joined first authorship.

Specialty section: This article was submitted to Marine Fisheries, Aquaculture and

Living Resources,

a section of the journal Frontiers in Marine Science

Received: 21 January 2016 Accepted: 05 April 2016 Published: 22 April 2016

Citation:

Schubert P, Vogt L, Eder K, Hauffe T and Wilke $T$ (2016) Effects of Feed

Species and HUFA Composition on Survival and Growth of the Longsnout Seahorse (Hippocampus reidi). Front. Mar. Sci. 3:53. doi: 10.3389/fmars.2016.00053

\author{
Patrick Schubert ${ }^{1 \star t}$, Lena Vogt ${ }^{1 \dagger}$, Klaus Eder ${ }^{2}$, Torsten Hauffe ${ }^{1}$ and Thomas Wilke ${ }^{1}$ \\ ${ }^{1}$ Department of Animal Ecology and Systematics, Justus Liebig University Giessen, Giessen, Germany, ${ }^{2}$ Department of \\ Animal Nutrition and Nutritional Physiology, Justus Liebig University Giessen, Giessen, Germany
}

Globally, wild seahorse populations are threatened due to, habitat destruction and unsustainable human exploitation among others. Furthermore, aquaculture-based mass-scale rearing is still uncommon due to the low survival rates of seahorse juveniles and exceptionally high feed costs. Previous studies have demonstrated the importance of both highly unsaturated fatty acid (HUFA) supplies and a copepod-based rearing for seahorse survival and growth. As the latter is expensive, the question arises as to how high survival rates of seahorse juveniles can be assured under low- to moderate-cost feed regimes. In particular, it remains unknown whether the diet species or their dietary HUFA profiles determine the successful development of seahorse fry. Therefore, the aims of this study were to assess the dependence of growth and survival rates of Hippocampus reidi brood on the animal feed and to infer the impact of feed species vs. dietary HUFA profiles on juvenile growth. A nutrition experiment was conducted where juveniles were treated either with enriched Artemia nauplii (low-cost diet Art) or with a mixed diet of Artemia and copepods (moderate-cost diet Art/Cop). Larval survival and growth were analyzed using Cox proportional-hazard and linear mixed-effect model analyses. We found that (i) both diets enabled good survival, (ii) diet Art/Cop resulted in superior weight and height growth, and (iii) the differential effects of diets Art/Cop and Art cannot be explained by their different HUFA compositions alone. From an economical point of view, our findings of high survival rates and relatively high growth rates with the medium-cost treatment Art/Cop may open new possibilities for the large-scale rearing of seahorses. Even the application of a low-cost Art diet might be appropriate for seahorse aquacultures as both survival and growth rates are only marginally lower compared to the former diet.

Keywords: aquaculture, Artemia, copepods, diet, highly unsaturated fatty acid, DHA, Eurytemora

\section{INTRODUCTION}

Globally, wild seahorse populations are threatened due to alteration and destruction of habitats as well as unsustainable exploitation, such as excessive usage for traditional medicine, decoration, and ornamental purposes (Vincent, 1996; Wabnitz et al., 2003; Evanson et al., 2011; Vincent et al., 2011). As a result, wild seahorse populations are in decline and the entire genus Hippocampus is listed in Appendix II of the Convention on International Trade in Endangered Species of Wild Fauna and Flora (CITES, 2004). 
In order to meet the increasing demand for seahorses and to stabilize wild populations, currently 13 species, including Hippocampus reidi, are cultured for ornamental trade. However, mass-scale rearing is still rare and commercial aquaculture for traditional medicine purposes is non-existent (Koldewey and Martin-Smith, 2010). This is mainly due to two reasons; (i) the low survival rates of seahorse juveniles (e.g., Woods, 2003; Foster and Vincent, 2004; Olivotto et al., 2011), and (ii) exceptionally high feed costs (e.g., Lavens and Sorgeloos, 1996).

Low survival rates arise from the sensitivity of seahorses to housing conditions, the vulnerability to diseases (like vibriosis and gas bubble disease) and special nutritional demands (Koldewey, 2005; Koldewey and Martin-Smith, 2010; Palma et al., 2013; Corse et al., 2014). The supply of actively moving zooplankton of adequate size is a critical factor in seahorse culture (Lavens and Sorgeloos, 1996; Wittenrich, 2007; Olivotto et al., 2011). Additionally, seahorses are characterized by a relatively inefficient digestion system due to an underdeveloped stomach (Tipton and Bell, 1988; Kumaravel et al., 2010) and a short digestive tract transit time (Koldewey, 2005; Murugan et al., 2009; Deobagkar et al., 2012). Therefore, they possess no significant fat reserves and are forced to have a high daily food intake (Koldewey, 2005; Wittenrich, 2007). Particularly during the first days of life, the availability of high-quality prey organisms in appropriate amounts determines survival and growth rates (Sheng et al., 2007). Moreover, the availability of the highly unsaturated fatty acids (HUFAs) arachidonic acid (AA, C20:4n6), eicosapentaenoic acid (EPA, C20:5n-3), and docosahexaenoic acid (DHA, C22:6n-3) is important for the health of marine fish larvae (Bell et al., 1986; Watanabe, 1993; Cahu et al., 2003; De Silva et al., 2012). In fact, Chang and Southgate (2001) found a significant correlation between mean survival of Hippocampus spp. juveniles and dietary n3-HUFA, EPA, and DHA contents.

Exceptionally high feed costs are mainly associated with the culture of feed species. Several authors (e.g., Payne and Rippingale, 2000; Olivotto et al., 2008; Celino et al., 2012; Willadino et al., 2012) have indicated relatively high survival and growth rates for seahorse juveniles reared with copepod diets. However, copepod-based rearing is expensive due to fluctuating survival and reproduction rates within copepod cultures (Lavens and Sorgeloos, 1996; Kaminski et al., 2014). Therefore, seahorse larval feeding protocols are commonly based on a mixed diet of rotifers, copepods, and/or strains of the brine shrimp Artemia spp. However, as Artemia nauplii display a deficiency in HUFAs (Sorgeloos et al., 2001; Otero-Ferrer et al., 2010), they are usually enriched by incubation in special HUFA solutions (Job et al., 2002; Woods, 2003; Wong and Benzie, 2003; Palma et al., 2013).

Integrating the two sets of problems outlined above, the question arises as to how high survival rates of seahorse juveniles can be assured with low to moderate feed costs. In particular, it would be useful to know whether the diet species or their dietary HUFA profiles determine the successful development of seahorse larvae (sensu Payne and Rippingale, 2000). In order to assess the dependence of growth and survival rates of $H$. reidi juveniles on low- to moderate-cost animal feeds and to quantify the impact of feed species vs. dietary HUFA profiles on the juvenile's survival and growth, we conducted a nutrition experiment under fully controlled lab conditions. The juveniles of some Hippocampus species, such as $H$. reidi, are relatively small and not able to feed on enriched Artemia nauplii within their first days of life (Olivotto et al., 2008). Therefore, the experiment started at an age of 8 days, when the individuals presumably had exhausted their internal fatty acid resources, but were large enough to pray on enriched Artemia (Sheng et al., 2007). It lasted for 3 weeks, until a point in time in which survival curves in juvenile seahorses tend to stabilize (see Woods, 2000; Wittenrich, 2007; Olivotto et al., 2008). During this period, individuals were treated either with enriched Artemia nauplii (low-cost diet Art) or with a mixed diet of Artemia and copepods (moderate-cost diet Art/Cop) with known HUFA profiles.

The specific goals of our study were:

(1) To assess the differential effects of the two feeds on survival rates of juvenile seahorses using survival analyses based on a Cox proportional-hazard model.

(2) To use linear mixed-effect model analyses to infer the effects of the two feeds on the height growth of specimens.

(3) To empirically assess the impact of dietary HUFA profiles of feed species on the development of juvenile seahorses.

(4) To discuss the influence of HUFA profiles vs. feed species.

This study improves our understanding of the nutritional demands of seahorse fry. It may also contribute to the discussion on how seahorse rearing can be made more cost-efficient toward the establishment of mass-production aquaculture facilities by testing the performance of low- to moderate cost feed species.

\section{MATERIALS AND METHODS}

\section{Broodstock}

To avoid a potentially bias of farmed seahorses due to pre-adaptions to artificial feed, we aimed at utilizing wildcaught animals for our nutritional experiments. However, both ethical concerns as well as national and international animal experimentation acts prevented us from using a large number of captured breeding pairs for our study. Therefore, two wildcaught and heavily pregnant $H$. reidi males from the Frankfurt Zoo (Germany) were transferred to the animal facilities at Justus Liebig University Giessen (Germany). The seahorses were held together in a 125 L broodstock tank, connected to a closed watersystem equipped with a delivery pump for water flow support (168 L $\cdot \mathrm{h}^{-1}$ per tank), protein skimmer, UV-filter, phosphate filter, and ozonizer. Seawater was prepared from reverse osmotic water and artificial sea salt (Reef Crystals, Sarrebourg, France) at the following conditions: Salinity $32-33 \mathrm{~g} \cdot \mathrm{L}^{-1}$, temperature $27^{\circ} \mathrm{C}$, alkalinity $107.09 \mathrm{mg} \cdot \mathrm{L}^{-1}, \mathrm{CaCO}_{3}, \mathrm{PO}_{4}^{3-}, \mathrm{NO}_{3}^{-}$and $\mathrm{NO}_{2}^{-}$ at $0.35 \mathrm{mg} \cdot \mathrm{L}^{-1}, 0-10 \mathrm{mg} \cdot \mathrm{L}^{-1}$, and $0 \mathrm{mg} \cdot \mathrm{L}^{-1}$, respectively. The light/darkness period (L/D) was 14L/10D (LED, $30 \mathrm{~W}$ ). Two air supplies provided ancillary oxygenation, and PVC rods and plastic plants served as holdfasts for the animals. The animals were fed five times a day to satiation with frozen "Kristallgarnelen" (Ascetes sp., Zooschatz, Berlin, Germany) and, frozen and live mysid shrimps (Fischfutter Etzbach, Schleiden, Germany), analogous to the feeding regimen at the Frankfurt Zoo. Feces and detritus were siphoned daily. Two days after 
acclimatization, one of the males gave birth to its brood $(N=$ 976), which was used for subsequent experiments.

\section{Diet Preparation \\ Copepods}

A copepod stock culture (Eurytemora affinis, Zooschatz, Berlin, Germany) was maintained at $18^{\circ} \mathrm{C}$ in a plastic tank $(80 \times 60 \times 10 \mathrm{~cm})$ under medium aeration and the following conditions: Salinity 32-33 $\mathrm{g} \cdot \mathrm{L}^{-1}, \mathrm{PO}_{4}^{3-}, \mathrm{NH}_{3}$, and $\mathrm{NO}_{2}^{-}$ approaching $0.0 \mathrm{mg} \cdot \mathrm{L}^{-1}$. The tank was illuminated at a period of 11L/13D (standard light bulb, $30 \mathrm{~W}$ ). Siphoning of food rests and feces, complete water exchanges, and monitoring of water parameters were performed regularly. Copepods were fed daily with an algae solution (Isochrysis galbana, Nannochloropsis oculata, Rhodomonas baltica, and Tetraselmis chuii) and with Spirulina (Ocean Star International, Snowville, USA) ad-libitum. Copepod feeding took their size/stage into account. Thus, naupliar stages (50-90 $\mu \mathrm{m})$ were divided from copepodite and adult stages $(150-500 \mu \mathrm{m})$ by using sieves of different mesh size $(35,65$, and $120 \mu \mathrm{m})$, and fed accordingly.

Juvenile seahorses were weaned to live copepods (Copalive) on day 8. To further minimize costs and efforts, we replaced Copalive with commercially available frozen copepods (Cop frost, "Cyclops Vitamin plus," Eurytemora affinis, Zooschatz, Berlin, Germany) on day 21.

\section{Artemia}

"AF 430 Artemia" cysts (Art $t_{A F}$, Inve Technologies, Dendermonde, Belgium), "Platinum Artemia" (Art platinum, Argent Labs, Redmond, USA), and "common Artemia" cysts

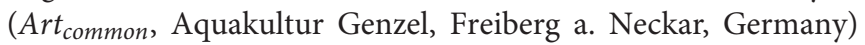
were hatched in self-made acrylic glass reactors. Constant illumination was provided by a standard light bulb (40 W). Hatching was initiated for $A r t_{A F}$ and Art $t_{\text {platinum }}$ cysts daily at 5 p.m. ( $1 \mathrm{~g}$ cysts per $1.5 \mathrm{~L}$ water) and for $A r t_{\text {common }}$ cysts at 9 a.m. ( $22 \mathrm{~g}$ cysts per $3 \mathrm{~L}$ water). Artemia enrichment was accomplished by using Selco S.presso (Inve Technologies; $2 \times 500$ ppm S.presso per $0.75 \mathrm{~L}$ water containing nauplii (npl) densities of up to 400 Artemia npl. $\mathrm{mL}^{-1}$, application at 0 and $10 \mathrm{~h}$ ) for $24 \mathrm{~h}$ at $25^{\circ} \mathrm{C}$ under sufficient aeration. Prior to diet application, all animal feeds were rinsed with fresh water.
Thirty minutes after diet-application, excess food organisms were removed with self-made filtering equipment to prevent overfeeding and to ensure an optimal digestion and assimilation of the nutrients.

\section{Experimental Design}

$H$. reidi juveniles were reared in self-made $8.5 \mathrm{~L}$ acrylic glass rearing tanks (Figure 1), constructed according to the kreisel principle (Wittenrich, 2007). Saltwater conditions were as follows: Salinity 32-33\%, moderate circular water current (circa two air bubbles per second), water temperature $24^{\circ} \mathrm{C}$, and $\mathrm{PO}_{4}^{3-}, \mathrm{NH}_{3}$ and $\mathrm{NO}_{2}^{-}$concentrations all approaching 0.0 $\mathrm{mg} \cdot \mathrm{L}^{-1}$. The tanks were illuminated (LED, $30 \mathrm{~W}$, length $120 \mathrm{~cm}$ ) circa $30 \mathrm{~cm}$ from above with an initial photoperiod of 23L/1D, as recommended by Wittenrich (2007) and Olivotto et al. (2008). The period was successively reduced by $1 \mathrm{~h}$ a day until 15L/9D. PVC rods and plastic plants were added as holdfasts after juveniles had finished their pelagic stage. An algae solution (consisting of I. galbana, N. oculata, R. baltica, and T. chuii) was added to the rearing tanks (as recommended by Wittenrich, 2007). Daily, a 25\% water exchange was performed, combined with siphoning feces and food remains. For hygiene maintenance, rearing tanks and equipment were disinfected regularly.

On the day of birth (day 1), all individuals of the brood $(N=976)$ were randomly distributed to twelve rearing tanks. At the beginning of the actual feeding experiment (day 8), the remaining seahorse juveniles $(n=360)$ were transferred to six separate rearing tanks with 60 individuals each (for experimental setup see Figure 1) and subjected to the diet-specific treatments.

During the pre-study period (days 1-7), all juveniles were fed with freshly hatched Artemia nauplii (430-450 $\mu \mathrm{m}$; Instar I nauplii; according to Treece, 2000) as follows: Days 1-2 Art $t_{\text {platinum }}$, days 3-5 Art $t_{\text {platinum }}$, and $A r t_{A F}$, days 5-7 enriched

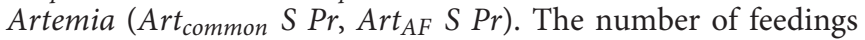
ranged from 3-8 times a day ad-libitum, at a food density of $0.5-1$ $\mathrm{npl} \cdot \mathrm{mL}^{-1}$, which showed the best results in preliminary studies.

For the main feeding experiment (days 8-30), one part of the animals were fed with Art, the other with Art/Cop. Treatment Art comprised enriched Artemia nauplii Art common $S \operatorname{Pr}$ and $A r t_{A F}$ $S P r$ at a ratio of 1:1 (naupliar stages Instar II and III at a size of 600-700 $\mu \mathrm{m}$; according to Treece, 2000); treatment Art/Cop a

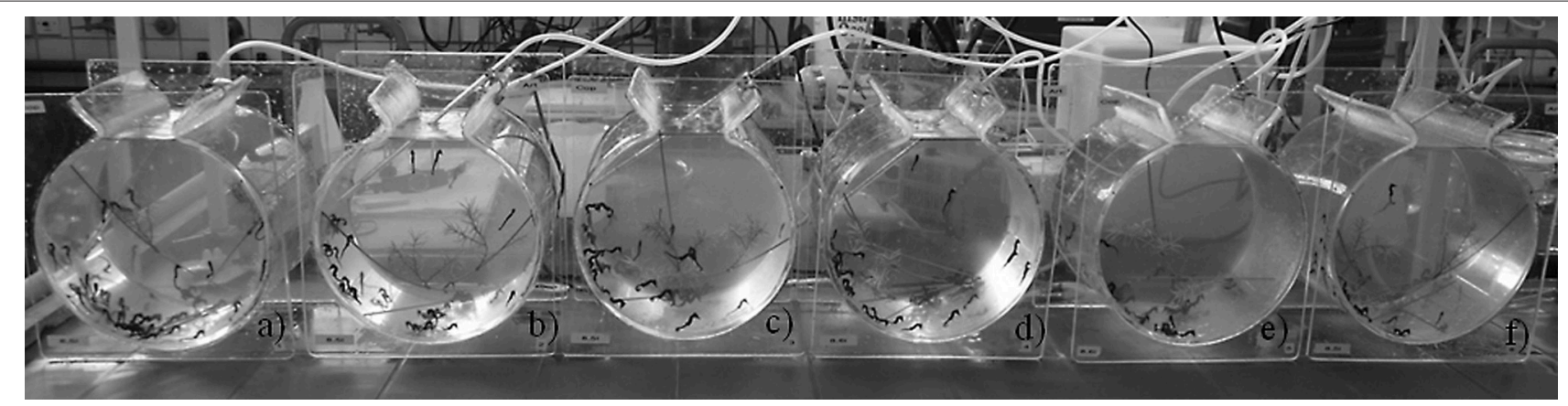

FIGURE 1 | Experimental setup for survival and growth analyses of Hippocampus reidi. (a) Diet Art/Cop 1, (b) diet Art 1, (c) diet Art/Cop 2, (d) diet Art 2, (e) $\operatorname{diet}$ Art/Cop 3, (f) diet Art 3. 
mixture of Art as well as living and frozen copepods at a ratio of 3/4:1/8:1/8 (copepod naupliar and adult stages at a size of 50-90 $\mu \mathrm{m}$ and 150-500 $\mu \mathrm{m}$, respectively; according to Wittenrich, 2007).

Juveniles were fed four times a day ( 9 a.m., 12 a.m., 2 p.m., 5 p.m.) at a density of $1-5 \mathrm{npl} \cdot \mathrm{mL}^{-1}$ (days 8-19) and 5-9 npl $\cdot \mathrm{mL}^{-1}$ (days 20-30), respectively.

\section{Growth and Survival Data Sampling}

Seahorse survival within each tank was recorded daily. On days $8,13,22$, and 31 , individual heights (distance from top of the head to the tip of the out-stretched tail, sensu Lourie et al., 2004) of the juveniles were measured. To minimize stress, this was done when the seahorses were removed from the tanks during regular disinfections. Accordingly, all specimens of a particular tank were transferred for approximately $1 \mathrm{~min}$ into a large Petri dish (diameter: $20 \mathrm{~cm}$ ) filled with $10-15 \mathrm{~mm}$ seawater, laterally positioned, and jointly photographed without flash light. Immediately after this procedure, the specimens were transferred back into the tank. Photographs were analyzed using the open source scalable vector graphics editor Inkscape 0.48.4.1 (http:// de.softonic.com/s/download-inkscape-0.48.4.1).

In contrast to height measurements, individual weight determinations of juveniles might result in high stress levels (e.g., convoluted bodies would need to be separated), frequently leading to death. To minimize handling effects, the weight was therefore only recorded at the end of the experiment (day 31) by weighting all juveniles per tank together. Thus, only mean values, without standard deviations, per tank are available for weight.

\section{Survival Analyses}

Estimations of the effect of diet on mortality after the prestudy period were established using a semi-parametric Cox proportional-hazard regression model (Cox, 1972). This models how the probability of a mortality event (i.e., risk or hazard) changes by one or more covariates (e.g., treatment). The model is semi-parametric, which means any variation of the baseline hazard over time is already included in the model and the influence of the covariates on mortality is, at any point in time, proportional to the baseline risk. Lifetime of $H$. reidi specimens were right censored. This means events of death were recorded, but the model was also informed about individuals surviving the end of the experiment. The rearing tanks were specified as statistically non-independent groups of observations. Our model was fitted using the survival package version 2.37-7 (Therneau, 2014) for the R statistical environment (version 3.0.2; R Core Team, 2014).

\section{Growth Analyses}

Given that weight data could only be generated without standard deviations, these data were not subjected to subsequent statistical analyses.

However, for the height data, the differential growth effects were jointly analyzed utilizing a single linear mixed-effect model (Fisher, 1935 in Sahai and Ageel, 2000).

This method was chosen because the measurements of seahorse heights within the six rearing tanks were repeated over time. Ignoring such a design might bias statistical inference due to pseudoreplications. The model was constructed with diet, time since beginning of the experiment and a quadratic term of time, and their interaction, as fixed factors. As random part of the mixed-effect regression model, time was nested in tanks, thus it was controlled for repeated measures and accounted for among aquarium and time variances in seahorse height by individual intercepts in the model. Because pre-tests showed a high correlation among the fixed effects estimates, the variable time was centered by subtracting its mean. The equation form of the model is given as: height ${ }_{i}=\beta$ diet $_{i}+\beta$ time $_{i}+\beta$ time $_{i}^{2}+$ $\beta$ diet $_{\mathrm{i}} \mathrm{x}_{\text {time }_{\mathrm{i}}}+\beta$ diet $_{i} \mathrm{x}$ time $_{\mathrm{i}}^{2}+\mathrm{u}_{\mathrm{i}} \mathrm{Z}_{\mathrm{i}}+\varepsilon_{\mathrm{i}}$; where i denotes the individual observation, $\beta$ the regression coefficients of the fixed effects, $\mathrm{x}$ the interaction between fixed effects, $\mathrm{uZ}$ the individual intercepts of the random effects tank and time, and $\varepsilon$ the residual. The linear mixed-effect model was fitted by maximizing the loglikelihood utilizing the R package nlme 3.1-113 (Pinheiro et al., 2014). According to Johnson and Omland (2004), we used the Akaike information criterion (AICc) for information-theoretic model selection by comparing the full model with simplified ones (i.e., omitting either interaction, time, diet, or all predictors).

\section{Fatty Acid Analyses of Animal Feeds}

Fresh samples of different animal feeds (described above) were freeze-dried overnight under vacuum $\left(-30^{\circ} \mathrm{C}\right.$, Gamma 1-20, Martin Christ $\mathrm{GmbH}$, Osterode, Germany) and then treated with hexane-isopropyl (3:2, v/v) (Hara and Radin, 1978; Eder and Kirchgessner, 1994) on a lab shaker (GFL 3015, Burgwedel, Germany) for $18 \mathrm{~h}$ at room temperature. Subsequently, the fluid extract was boiled down $\left(37^{\circ} \mathrm{C}\right)$, whilst being nitrogenated. Fatty acids were methylizated by trimethylsulfonium hydroxide (Butte, 1983) and fatty acid methyl esters (FAMEs) were analyzed using a gas chromatograph (Clarus 580, PerkinElmer, Waltham, USA) coupled with a flame ionization detector (FID). For chromatographic fractionation, a capillary column (FFAPOptima Plus, $50 \mathrm{~m}$ column length, $0.25 \mu \mathrm{m}$ internal diameter, $0.2 \mu \mathrm{m}$ film diameter, Macherey-Nagel, Düren, Germany) and helium, as carrier gas (flow rate of $1.2 \mathrm{~mL} \cdot \mathrm{min}^{-1}$ ), were used. Oven temperature was $140^{\circ} \mathrm{C}$ for $2 \mathrm{~min}$., then increased to $200^{\circ} \mathrm{C}$ for $15 \mathrm{~min}$. and further increased to $240^{\circ} \mathrm{C}$ for $25 \mathrm{~min}$. Both the split injector (1:20) and the FID were set to $260^{\circ} \mathrm{C}$. A FAME standard solution (18919-1 AMP Supelco, SigmaAldrich, Deisenhofen, Germany) was used for identification and quantification of individual FAMEs in the feed samples.

\section{RESULTS}

\section{Survival Rates}

After the first 4 days since birth seahorse survival (initially $N=$ 976) was $95 \%$. It sharply declined to about $37 \%$ between days 5 and 7. During the main study (days $8-31$ ) it plateaued with an overall survival rate of approximated $32 \%(n=311)$. Juveniles of treatment Art/Cop revealed slightly higher survivorship, mean height, and weight growths than juveniles of treatment Art. Treatment and replicate-specific survival rates, and treatmentspecific height and weight gains are shown in Table $\mathbf{1}$.

With a hazard ratio of 1.34 , the fitted survival model also showed a higher probability of survival for juveniles of treatment 
TABLE 1 | Survival rates, mean heights, and mean weights of Hippocampus reidi specimens of treatments Art/Cop (replicates 1-3) and Art (replicates $1-3)$ on days $8(n=360), 13(n=326), 22(n=322)$, and 31 $(n=320)$.

\begin{tabular}{|c|c|c|c|c|c|}
\hline \multirow[t]{2}{*}{ Treatment } & \multirow[t]{2}{*}{ Replicate } & \multicolumn{4}{|c|}{ Survival rate (\%) } \\
\hline & & Day 8 & Day 13 & Day 22 & Day 31 \\
\hline \multirow[t]{3}{*}{ Art/Cop } & 1 & 100 & 97 & 95 & 95 \\
\hline & 2 & 100 & 80 & 78 & 78 \\
\hline & 3 & 100 & 97 & 95 & 93 \\
\hline \multirow[t]{4}{*}{ Art } & 1 & 100 & 87 & 87 & 87 \\
\hline & 2 & 100 & 88 & 85 & 83 \\
\hline & 3 & 100 & 95 & 95 & 95 \\
\hline & & \multicolumn{4}{|c|}{ Mean height \pm SD $(\mathrm{mm})$} \\
\hline \multirow[t]{3}{*}{ Art/Cop } & 1 & $11.4 \pm 1.4$ & $16.3 \pm 2.5$ & $22.5 \pm 2.9$ & $28.8 \pm 2.7$ \\
\hline & 2 & $11.1 \pm 1.8$ & $16.8 \pm 2.8$ & $23.5 \pm 3.4$ & $30.1 \pm 2.9$ \\
\hline & 3 & $11.5 \pm 1.5$ & $15.9 \pm 2.5$ & $23.2 \pm 2.3$ & $30.2 \pm 2.3$ \\
\hline \multirow[t]{4}{*}{ Art } & 1 & $11.0 \pm 1.7$ & $15.4 \pm 1.9$ & $20.5 \pm 3.1$ & $27.1 \pm 3.3$ \\
\hline & 2 & $11.7 \pm 1.7$ & $14.9 \pm 2.2$ & $22.4 \pm 2.2$ & $27.2 \pm 3.6$ \\
\hline & 3 & $11.4 \pm 1.6$ & $15.2 \pm 2.6$ & $21.2 \pm 3.3$ & $26.8 \pm 4.0$ \\
\hline & & \multicolumn{4}{|c|}{ Mean weight (mg) } \\
\hline \multirow[t]{3}{*}{ Art/Cop } & 1 & $\mathrm{n} / \mathrm{a}$ & $\mathrm{n} / \mathrm{a}$ & $\mathrm{n} / \mathrm{a}$ & 158.46 \\
\hline & 2 & $\mathrm{n} / \mathrm{a}$ & $\mathrm{n} / \mathrm{a}$ & $\mathrm{n} / \mathrm{a}$ & 99.7 \\
\hline & 3 & $\mathrm{n} / \mathrm{a}$ & $\mathrm{n} / \mathrm{a}$ & $\mathrm{n} / \mathrm{a}$ & 90.6 \\
\hline \multirow[t]{3}{*}{ Art } & 1 & $\mathrm{n} / \mathrm{a}$ & $\mathrm{n} / \mathrm{a}$ & $\mathrm{n} / \mathrm{a}$ & 101.6 \\
\hline & 2 & $\mathrm{n} / \mathrm{a}$ & $\mathrm{n} / \mathrm{a}$ & $\mathrm{n} / \mathrm{a}$ & 77.6 \\
\hline & 3 & $\mathrm{n} / \mathrm{a}$ & $\mathrm{n} / \mathrm{a}$ & $\mathrm{n} / \mathrm{a}$ & 71.7 \\
\hline
\end{tabular}

Art/Cop, however the difference between the two treatments was not significant $(z=-0.758, P=0.449)$.

\section{Weight-Growth Values}

At the end of the experiment, juveniles of treatment Art/Cop showed greater mean weight values than juveniles of treatment Art. Accordingly, juveniles of treatment Art/Cop displayed mean weight values of $90.64-158.46 \mathrm{mg}$, whereas juveniles of treatment Art showed values of 71.66-101.61 mg (Table 1).

\section{Height-Growth Rates}

At the beginning of the main experiment (day 8), juveniles where similar in height. However, until the end of the experiment (day 31), juveniles of treatment Art/Cop showed a greater height increase than juveniles of treatment Art (see Figure 2).

Model selection identified the linear-mixed effect regression as the model with the highest fit $(\mathrm{AICc}=6320.4)$ that included all terms, except the interaction of treatment with the quadratic term of time. All remaining predictors (diet, time, a quadratic term of time, and the interaction between time and treatment) were significant (Table 2). At the end of the experiment, $H$. reidi specimens of treatment Art/Cop were, on average, $2.7 \mathrm{~mm}$

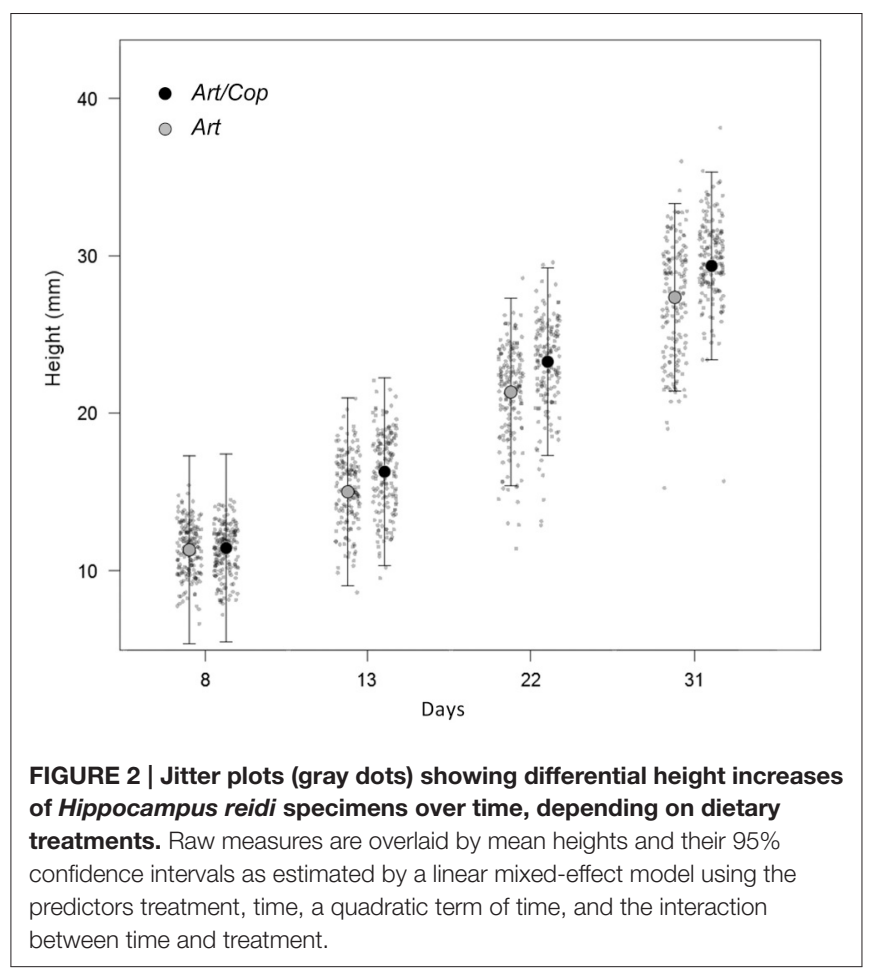

TABLE 2 | Linear mixed-effect explaining height of Hippocampus reidi larvae depending on two dietary treatments.

\begin{tabular}{lcccc}
\hline & $\boldsymbol{\beta}$ & SE & DF & $\boldsymbol{T}$ \\
\hline Intercept & 18.892 & 0.185 & 1304 & $102.293^{\star * *}$ \\
Treatment Art/Cop & 1.324 & 0.190 & 4 & $6.951^{\text {** }}$ \\
Time & 0.694 & 0.015 & 15 & $45.575^{\text {***}}$ \\
Time $^{2}$ & -0.005 & 0.002 & 15 & $-3.114^{\star *}$ \\
Interaction Treatment & 0.109 & 0.021 & 15 & $5.309^{* * *}$ \\
Art/Cop $\times$ Time & & & & \\
\hline
\end{tabular}

The regression coefficients $\beta$ and their standard errors (SE) are given for all five predictors. Their significance was inferred based on the $t$-statistic, with reduced degrees of freedom (DF) because of the repeated measurement of height within the same tanks and time.

${ }^{\star * \star} P \leq 0.001,{ }^{* \star} P \leq 0.01,{ }^{*} P \leq 0.05$.

$(S E=0.25 \mathrm{~mm}$ ) larger than of treatment Art (Figure 2). The negative regression coefficient of the quadratic term of time $(-0.005 \pm 0.002)$ indicates a non-linear reduction in growth rates. However, the coefficient of the interaction treatment Art/Cop with the term time suggests that the diet-specific difference became more pronounced over time.

\section{Fatty Acid Profiles of Animal Feeds}

The fatty acid profiles of the animal feeds differed strongly between Art and Art/Cop in respect to main fatty acid and HUFA compositions (Table 3). In summary, unenriched Artemia $\left(\right.$ Art $\left._{\text {platinum }}, A r t_{A F}\right)$ contained the highest AA and EPA contents of all animal feeds but negligible DHA. In contrast, Copalive and Cop frost featured low AA and EPA levels and a medium level of DHA. Enriched Artemia nauplii (Art common $S$ Pr, Art $A F S P r$ ) 
TABLE 3 | Percentages and ratios of selected fatty acids within different animal feeds of Hippocampus reidi.

\begin{tabular}{|c|c|c|c|c|c|c|c|c|c|}
\hline Food & Main fatty acid (\%) & AA (\%) & EPA (\%) & DHA (\%) & HUFA $^{a}(\%)$ & n-3 (\%) & n-6 (\%) & DHA: EPA & $n-3: n-6$ \\
\hline Copalive & C 16:0 (28.14) & 0.95 & 5.11 & 6.74 & 12.80 & 11.85 & 0.95 & 1.32 & 12.50 \\
\hline Copfrost & C 16:0 (37.32) & 0.47 & 6.85 & 9.53 & 18.23 & 17.77 & 0.47 & 1.39 & 38.21 \\
\hline Art common $\mathrm{S} P r$ & C 18:1 n9 (17.39) & 1.81 & 11.61 & 12.49 & 26.09 & 24.28 & 1.81 & 1.08 & 13.42 \\
\hline $\operatorname{Art}_{A F} S P r$ & C 20:5 n3 (16.62) & 3.79 & 16.62 & 9.30 & 29.74 & 25.96 & 3.79 & 0.56 & 6.85 \\
\hline Artcommon & C 18:1 n9 (18.19) & 1.33 & 12.16 & 0 & 13.71 & 12.38 & 1.33 & 0 & 9.34 \\
\hline $\operatorname{Art}_{A F}$ & C 20:5 n3 (20.13) & 4.15 & 20.13 & 0.15 & 24.51 & 20.37 & 4.15 & 0.01 & 4.91 \\
\hline Art $_{\text {platinum }}$ & C 20:5 n3 (18.96) & 4.49 & 18.96 & 0.17 & 23.70 & 19.21 & 4.49 & 0.01 & 4.28 \\
\hline
\end{tabular}

a HUFA: percentages of DHA, EPA, AA, and ETA (20:3 n-3).

showed the highest DHA and HUFA contents. Cop frost revealed the highest DHA:EPA and n-3:n-6 ratios. The calculated relative percentages and ratios of HUFAs within diets Art and Art/Cop revealed that diet Art contained slightly higher DHA, EPA, and AA amounts than diet Art/Cop (Table 4).

\section{DISCUSSION}

The main findings of this study are: (i) Both the diet based on enriched Artemia nauplii and living/frozen copepods (diet Art/Cop), and the diet solely based on enriched Artemia nauplii (diet $A r t$ ), provide sufficient nutrients and fatty acids to enable good survival and growth of juvenile seahorses. However, diet Art/Cop resulted in superior weight and height growth in comparison to diet Art. (ii) The difference in height growth between treatments increased over time with juveniles of treatment Art/Cop showing a significantly increased final height. (iii) Due to higher amounts of HUFAs in diet Art, the superior juvenile survival and growth performance with diet Art/Cop cannot be explained by different HUFA compositions alone.

\section{General Nutritional Problems in Juvenile Seahorses}

Faleiro and Narciso (2010) found seahorse embryos having a high metabolic turnover of DHA, AA, EPA, and C 16:0 (Palmitic acid) resulting in a low amount of lipids and largely depleted HUFA contents in newborn juveniles. They are completely dependent on exogenous food resources, as marine fishes are neither able to synthesize DHA de novo or to convert it from precursors in sufficient amount (Sargent et al., 1995, 2002). DHA, EPA, and AA play important roles as constituents in biomembranes and in immune response (Watanabe, 1993; Sargent et al., 1995; Ullrey, 1995; Mc Donald et al., 2002). Furthermore, an undersupply of DHA has been shown to cause malformation, slower growth, and enhanced mortality in fishes (Shields et al., 1999). In fact, Sheng et al. (2007) suggested that the high mortality of Hippocampus spp., generally observed during the first week of life, might be related to the exhaustion of the juvenile's fatty acid resources.

This is consistent with the results observed in this study, where a sharp decrease in survival rates could be seen within the first week. The effect might have been caused by the lack of sufficient DHA as mortality rates in our experiment decreased
TABLE 4 | Estimates of the relative HUFA percentages and ratios in the diets Art and Art/Cop.

\begin{tabular}{lrrrrr}
\hline Diet & AA & EPA & DHA & DHA: EPA & AA: EPA \\
\hline $2 \times$ Art $_{\text {AF } S P r}$ & 7.58 & 33.24 & 18.60 & & \\
$2 \times$ Art $_{\text {common }}$ & 3.62 & 23.22 & 24.98 & & \\
$S$ Pr & & & & & 0.20 \\
Diet Art & 11.20 & 56.46 & 43.58 & 0.77 & \\
$2 \times$ Cop alive $_{2 \times \text { Cop }_{\text {frost }}}$ & 1.90 & 10.22 & 13.48 & & \\
$2 \times$ Cop $_{\text {alive }}+$ & 0.94 & 13.70 & 18.70 & & \\
$2 \times$ Cop frost $_{\text {Diet Art/Cop }}^{2.84}$ & 23.92 & 32.18 & & 0.19 \\
\hline
\end{tabular}

The calculation is emanated from the fatty acid percentages listed in Table $\mathbf{3}$ and based

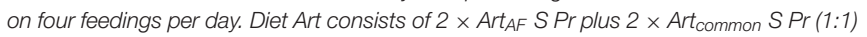

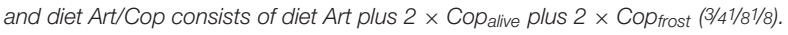

after applying enriched Artemia nauplii (abundant in DHA) starting at day 5 (data not shown here). Higher survival and growth rates as a result of the application of enriched Artemia nauplii were also reported by Chang and Southgate (2001), Quintas et al. (2010), Willadino et al. (2012), and Segade et al. (2014). Survival rates further stabilized after the application of diets Art and Art/Cop, which provided higher amounts of DHA as well as greater DHA:EPA and n-3:n-6 HUFA ratios than the former diets (Tables 3, 4).

\section{Differential Effect of the Two Feeds on Survival and Growth Rates}

The findings presented here show consistently higher survival rates as well as superior height and weight growth values in $H$. reidi specimens treated with feed Art/Cop compared to diet Art. These are in agreement with the findings of previous studies targeting other Hippocampus species (see Payne and Rippingale, 2000; Olivotto et al., 2008; Celino et al., 2012; Willadino et al., 2012). Payne and Rippingale (2000), for example, reported for $H$. subelongatus survival rates after day 14 of $>80 \%$ for specimens fed with copepods compared to $>40 \%$ for those fed with Artemia nauplii, and Job et al. (2002) noted survival rates for $H$. kuda ranging between 40 and $75 \%$, depending on type of enrichment. Moreover, Olivotto et al. (2008) showed a maximum survival rate after day 21 of $35 \%$ for $H$. reidi juveniles feeding on copepods 
compared to $20 \%$ for those reared with an Artemia nauplii diet. As suggested by Lavens and Sorgeloos (1996), the high costs of copepod cultures largely prevent the application of copepod species for large scale aquaculture of seahorses. Considering these economical constrains, our findings of high survival rates and relatively high growth rates with the moderate-cost treatment Art/Cop (in combination with the application of Cop frost starting from day 21) may open new possibilities for the large-scale rearing of seahorses. Even the application of a low-cost Art diet might be appropriate for seahorse aquaculture, as both survival and growth rates are only marginally lower compared to the former diet. In fact, our survival rates for both diets Art and Art/Cop where comparable or even higher than those reported by other authors for high-cost copepod diets (see Payne and Rippingale, 2000; Olivotto et al., 2008).

\section{Effects of Feed Species vs. HUFA Composition}

Given the findings discussed above, the question remains what causes the superior effect of a copepod diet. Is it the feed species or their differential dietary HUFA profiles?

Payne and Rippingale (2000) conducted a comparative study (copepods vs. enriched Artemia nauplii) to assess the effect of dietary fatty acid profiles on growth and survival rates of seahorse juveniles. They found significantly better survival, and increases in weight and length, in newborn $H$. subelongatus reared on copepod nauplii. Payne and Rippingale (2000) also showed that their copepod and Artemia feeds contained overall similar HUFA amounts, but that copepods had the fivefold DHA content (also see Evjemo and Olsen, 1997; Van der Meeren et al., 2008). This would indicate that the superior effect of the copepod feed might be largely due to its high DHA amount.

Though results of the present study also found superior survival and growth rates with a diet containing copepods (Art/Cop), it had, in contrast to the study of Payne and Rippingale (2000), both lower HUFA and DHA amounts than diet Art (see Table 3). Therefore, our results suggest that not HUFA (especially DHA) contents, but rather the feed species might be a determining factor for survival and growth of seahorse juveniles. This finding is in accordance with the results of several other studies showing the positive effect of copepods on survival and growth of seahorse juveniles and the copepods' nutritional superiority over Artemia nauplii under unenriched and even enriched conditions (see Gardner, 2003; Murugan et al., 2009; Celino et al., 2012; Willadino et al., 2012).

Biological parameters that might influence a consumer's nutrient/fatty acid assimilation capability, are digestibility and the availability fatty acid in feed organisms. For instance, gut and feces analyses of $H$. subelongatus and $H$. guttulatus have shown a better digestibility of copepods compared to enriched Artemia nauplii. The latter were found to be alive and intact within the feces of seahorses (Payne and Rippingale, 2000; Blanco, 2014; pers. observ.). The superior digestibility of copepods may be ascribed to the evolutionary adaptation of the digestive tract and enzymatic activity of seahorses to copepods, as they constitute a major part of the natural diet of most seahorse juveniles (Tipton and Bell, 1988; Castro et al., 2008). Additionally, Palma et al. (2013) found that $H$. guttulatus juveniles fed on enriched Artemia nauplii had a higher percentage of gas bubble infections compared to those fed on copepods. Delcroix et al. (2015) showed a strong correlation between diet and bacterial flora in marine fishes. In fact, the bacterial flora within the seahorses' gastrointestinal tract is competent of the enzymatic degradation of lipids, proteins, starch, cellulose, and xylan, whereby a high lipase activity was found (Deobagkar et al., 2012). The latter authors suggested that the modification of this bacterial flora could promote food digestion and fatty acid assimilation. Notwithstanding that the bacterial communities of the feed species used in the present study were not investigated, it could be possible that copepods contain an advantageous bacterial flora that is used by seahorses to enhance digestion. Furthermore, in copepods the fatty acid availability seems to be higher than in Artemia nauplii. For example, Bell and Sargent (2003) found copepods containing most of their HUFAs in the polar lipid fraction. In contrast, HUFAs in Artemia nauplii are mainly found in the neutral lipid fraction (Coutteau and Mourente, 1997). According to McKinnon et al. (2003), HUFAs in the polar lipid fraction are better available and utilizable than esterified HUFAs within triacylglycerols.

Other factors that might have contributed to the superior growth and survival of juveniles of treatment Art/Cop are HUFA ratios, proteins, and antioxidants. It was previously shown that the proper DHA:EPA ratio in favor of DHA plays an important role in fish larval development, growth and survival (Watanabe, 1993; Shields et al., 1999; Chang and Southgate, 2001). Additionally, EPA:AA ratios influence stress resistance and immune response because these fatty acids constitute precursors of pro- and anti-inflammatory eicosanoids (Ullrey, 1995; Mc Donald et al., 2002). Moreover, proteins and essential amino acids are important for the health and development of fishes by promoting the digestibility of fat, proteins, and starch (Nordrum et al., 2000).

\section{Outlook}

Our findings of high $H$. reidi survival rates under moderate- and low-cost feed treatments, and the importance of the feed species call for future studies to further improve seahorse aquacultures. Such studies should not only test the transferability of our results to other populations and species, they may also focus on enhancing diet compositions under an economical point of view. Furthermore, of interest are differences between Artemia nauplii and copepods in respect to their digestibility, fatty acid availability, protein and amino acid contents, and composition of bacterial communities. Moreover, there is considerable potential for improving the $H$. reidi feeding regime during the first days of life (i.e., until the juveniles reached a size to feed on enriched Artemia nauplii) in respect to DHA supplements.

\section{CONCLUSIONS}

Acknowledging a small sample size, this study suggests suitable husbandry conditions and feeding protocols for the rearing of $H$. reidi. Our results indicate that the availability of DHA largely 
determines the survival of juveniles during the first days of life. Furthermore, it was shown that not the dietary HUFA composition/content but the food species is the determining factor for growth. However, as a copepod-based rearing is costly, our results suggest a medium-cost feeding regimen for large-scale aquaculture that is based on enriched Artemia nauplii and a low amount of living or frozen copepods. Even a diet solely based on enriched Artemia nauplii may provide a suitable alternative under an economical point of view.

\section{AUTHOR CONTRIBUTIONS}

PS has done the conception of the work, interpretation of data, and part in drafting. LV carried out the major part of practical work, acquisition of data and a considerable part in drafting. KE was responsible for HUFA analysis and this part of the draft.

\section{REFERENCES}

Bell, J. G., and Sargent, J. R. (2003). Arachidonic acid in aquaculture feeds: current status and future opportunities. Aquaculture 218, 491-499. doi: 10.1016/S00448486(02)00370-8

Bell, M. V., Henderson, R. J., and Sargent, J. R. (1986). The role of polyunsaturated fatty acids in fish. Comp. Biochem. Physiol. B 83B, 711-719. doi: 10.1016/03050491(86)90135-5

Blanco, A. C. (2014). Rearing of the Seahorse Hippocampus guttulatus: Key Factors Involved in Growth and Survival. $\mathrm{PhD}$ Thesis. University of the Balearic Islands (UIB)

Butte, W. (1983). Rapid method for the determination of fatty acid profiles from fats and oils using trimethylsulfonium hydroxide for transesterification. $J$. Chromatogr. 261, 142-145. doi: 10.1016/S0021-9673(01)87931-0

Cahu, C., Zambonino Infante, J., and Takeuchi, T. (2003). Nutritional components affecting skeletal development in fish larvae. Aquaculture 227, 245-258. doi: 10.1016/S0044-8486(03)00507-6

Castro, A. L. D. C., Diniz, A. D. F., Martins, I. Z., Vendel, A. L., Oliveira, T. P. R. D., and Rosa, I. M. D. L. (2008). Assessing diet composition of seahorses in the wild using a non destructive method: Hippocampus reidi (Teleostei: Syngnathidae) as a study-case. Neotrop. Ichthyol. 6, 637-644. doi: $10.1590 /$ S1679-62252008000400012

Celino, F. T., Hilomen Garcia, G. V., and Norte Campos, A. G. C. D. (2012). Feeding selectivity of the seahorse, Hippocampus kuda (Bleeker), juveniles under laboratory conditions. Aquac. Res. 43, 1804-1815. doi: 10.1111/j.13652109.2011.02988.x

Chang, M., and Southgate, P. C. (2001). Effects of varying dietary fatty acid composition on growth and survival of seahorse, Hippocampus sp., juveniles. Aquar. Sci. Conserv. 3, 205-214. doi: 10.1023/A:1011363807074

CITES (2004). Notification to the Parties. Trade in Seahorses. Implementation of Decision 12.54. Available online at: http://www.cites.org/eng/notif/2004/033. pdf (Accessed: 26.08.2013)

Corse, E., Valladares, S., Planas, M., Chamorro, A., and Pintado, J. (2014). Analysis of the diet of the long-snouted seahorse Hippocampus guttulatus by 18 SrDNA amplification of prey in faeces. Aqua. Nutr. 21, 528-540. doi: $10.1111 /$ anu. 12189

Coutteau, P., and Mourente, G. (1997). Lipid classes and their content of n-3 highly unsaturated fatty acids (HUFA) in Artemia franciscana after hatching, HUFA-enrichment and subsequent starvation. Mar. Biol. 130, 81-91. doi: $10.1007 / \mathrm{s} 002270050227$

Cox, D. R. (1972). Models and life-tables regression. J. Roy. Statist. Soc. Ser. B 34, $187-220$.

Delcroix, J., Gatesoupe, F.-J., Desbruyères, E., Huelvan, C., Le Delliou, H., Le Gall, M.-M., et al. (2015). The effects of dietary marine protein hydrolysates on the development of sea bass larvae, Dicentrarchus labrax, and associated microbiota. Aqua. Nutr. 21, 98-104. doi: 10.1111/anu.12139
$\mathrm{TH}$ was responsible for data analysis and the respective part of the draft. TW interpretation of data, drafting and revising of the manuscript

\section{ACKNOWLEDGMENTS}

This study was supported by the DAAD Center of Excellence in Marine Sciences (CEMarin). Special thanks go to Thomas Tikatsch from the Frankfurt Zoo for the provision of the adult seahorses and to INVE ${ }^{\circledR}$ Technologies for supplying the different animal feeds (Artemia and enrichment). We thank Dr. Erika Most, Nicole Krämer, and Helmut Henzel (University of Giessen) for laboratory assistance. We also thank two anonymous referees and the editor for their useful comments on a previous version of the manuscript.

Deobagkar, D. D., Khandeparker, R., Sreepada, R. A., Sanaye, S. V., and Pawar, H. B. (2012). A study on bacteria associated with the intestinal tract of farmed yellow seahorse, Hippocampus kuda (Bleeker, 1852): characterization and extracellular enzymes. Aquac. Res. 43, 386-394. doi: 10.1111/j.1365-2109.2011. 02841.x

De Silva, S., Turchini, G., and Francis, D. (2012). "Nutrition," in Aquaculture: Farming Aquatic Animals and Plants, 2nd Edn., eds J. S. Lucas and P. C. Southgate (Chichester: Wiley-Blackwell), 164-187.

Eder, K., and Kirchgessner, M. (1994). The effect of zinc deficiency on erythrocyte membranelipids of force-fed rats receiving a diet containing coconut oil or fish oil. J. Trace Elem. Electrol. Health Dis. 8, 63-73.

Evanson, M., Foster, S. J., and Vincent, A. C. J. (2011). Tracking the International Trade Of Seahorses (Hippocampus Species) - The Importance of CITES. Fisheries Centre Research Reports, 19. Fisheries Centre, University of British Columbia, Vancouver, BC.

Evjemo, J. O., and Olsen, Y. (1997). Lipid and fatty acid content in cultivated live feed organisms compared to marine copepods. Hydrobiologia 358, 159-162. doi: 10.1023/A:1003196829109

Faleiro, F., and Narciso, L. (2010). Lipid dynamics during early development of Hippocampus guttulatus seahorses: searching for clues on fatty acid requirements. Aquaculture 307, 56-64. doi: 10.1016/j.aquaculture.2010. 07.005

Foster, S. J., and Vincent, A. C. J. (2004). Life history and ecology of seahorses: implications for conservation and management. J. Fish Biol. 65, 1-61 doi: 10.1111/j.0022-1112.2004.00429.x

Gardner, T. (2003). "The Copepod/Artemia tradeoff in the captive culture of Hippocampus erectus, a vulnerable species in Lower New York State," in Marine Ornamental Species: Collection, Culture and Conservation, eds J. C. Cato and C. L. Brown (Ames, IA: Blackwell Publishing Company), 297-303.

Hara, A., and Radin, N. S. (1978). Lipid extraction of tissues with a low-toxicity solvent. Anal. Biochem. 90, 420-426. doi: 10.1016/0003-2697(78)90046-5

Job, S. D., Do, H. H., Meeuwig, J. J., and Hall, H. J. (2002). Culturing the oceanic seahorse, Hippocampus kuda. Aquaculture 214, 333-341. doi: 10.1016/S00448486(02)00063-7

Johnson, J. B., and Omland, K. S. (2004). Model selection in ecology and evolution. Trends Ecol. Evol. 19, 101-108. doi: 10.1016/j.tree.2003.10.013

Kaminski, S. M., Bersano, J. G. F., and Freire, C. A. (2014). Euryhalinity of the estuarine copepod Pseudodiaptomus richardi and its high potential to be employed as live food in aquaculture. Aquaculture 224-225, 63-70. doi: 10.1016/j.aquaculture.2013.12.034

Koldewey, H. (2005). "Syngnathid husbandry in Public Aquariums 2005 Manual," in eds C. D. Bull and J. S. Mitchell (London: Project Seahorse). Available online at: http://www.intaquaforum.org/hg_FAI_Syngnathid05.pdf (Accessed: 26.08.2013).

Koldewey, H. J., and Martin-Smith, K. M. (2010). A global review of seahorse aquaculture. Aquaculture 302, 131-152. doi: 10.1016/j.aquaculture.2009.11.010 
Kumaravel, K., Ravichandran, S., and Balasubramanian, T. (2010). Variation in the gut micro flora of seahorses from indian waters. Internet J. Food Safety 12, 95-99. Available online at: http://www.internetjfs.org/articles/ijfsv12-14.pdf

Lavens, P., and Sorgeloos, P. (1996). Manual on the Production and Use of Live Food for Aquaculture, Vol. 361. Food and Agriculture Organization (FAO) Fisheries Technical paper. Available online at: ftp://ftp.fao.org/docrep/fao/003/w3732e/ w3732e00.pdf (Accessed 03.11.2015).

Lourie, S. A., Foster, S. J., Cooper, E. W. T., and Vincent, A. C. J. (2004). “A guide to the identification of Seahorses," in Project Seahorse and TRAFFIC North America (Washington, DC: University of British Columbia and World Wildlife Fund), 115. Available online at: http://www.traffic.org/species-reports/traffic_ species_fish29.pdf (Accessed: 26.08.2013)

Mc Donald, P., Edwards, R. A., Greenhalgh, J. F. D., and Morgan, C. A. (2002). Animal Nutrition, 6th Edn. Harlow: Prentice-Hall.

McKinnon, A. D., Duggan, S., Nichols, P. D., Rimmer, M. A., Semmens, G., and Robino, B. (2003). The potential of tropical paracalanid copepods as live feeds in aquaculture. Aquaculture 223, 89-106. doi: 10.1016/S0044-8486(03)00161-3

Murugan, A., Dhanya, S., Sreepada, R. A., Rajagopal, S., and Balasubramanian, T. (2009). Breeding and mass-scale rearing of three spotted seahorse, Hippocampus trimaculatus Leach under captive conditions. Aquaculture 290, 87-96. doi: 10.1016/j.aquaculture.2009.01.033

Nordrum, S., Krogdahl, Å., Røsjø, C., Olli, J. J., and Holm, H. (2000). Effects of methionine, cysteine and medium chain triglycerides on nutrient digestibility, absorption of amino acids along the intestinal tract and nutrient retention in Atlantic salmon (Salmo salar L.) under pair-feeding regime. Aquaculture 186, 341-360. doi: 10.1016/S0044-8486(99)00385-3

Olivotto, I., Avella, M. A., Sampaolesi, G., Piccinetti, C. C., Navarro Ruiz, P., and Carnevali, O. (2008). Breeding and rearing the longsnout seahorse Hippocampus reidi: rearing and feeding studies. Aquaculture 283, 92-96. doi: 10.1016/j.aquaculture.2008.06.018

Olivotto, I., Planas, M., Simões, N., Holt, G. J., Avella, M. A., and Calado, R. (2011). Advances in breeding and rearing marine ornamentals. J. World Aqua. Soc. 42, 2. doi: 10.1111/j.1749-7345.2011.00453.x

Otero-Ferrer, F., Molina, L., Socorro, J., Herrera, R., Fernández Palacios, H., and Soledad Izquierdo, M. (2010). Live prey first feeding regimes for short snouted seahorse Hippocampus hippocampus (Linnaeus, 1758) juveniles. Aquac. Res. 41, e8-e19. doi: 10.1111/j.1365-2109.2010.02505.x

Palma, J., Bureau, D. P., and Andrade, J. P. (2013). The effect of diet on ontogenic development of the digestive tract in juvenile reared long snout seahorse Hippocampus guttulatus. Fish. Physiol. Biochem. 40, 739-750. doi: 10.1007/s10695-013-9881-8

Payne, M. F., and Rippingale, R. J. (2000). Rearing West Australian seahorse, Hippocampus subelongatus, juveniles on copepod nauplii and enriched Artemia. Aquaculture 188, 353-361. doi: 10.1016/S0044-8486(00)00349-5

Pinheiro, J., Bates, D., DebRoy, S., and R Core Team (2014). nlme: Linear and Nonlinear Mixed Effects Models. Available online at: http://CRAN.R-project.org/package=nlme

Quintas, P., Planas, M., and Purser, G. J. (2010). "Biochemical composition and enzymatic activities in juveniles of the seahorse Hippocampus abdominalis and the effect of Artemia enrichment," in Aquaculture Europe, Vol. 10, (Porto: E. A. Society), 1053-1054.

R Core Team (2014). R: A language and Environment for Statistical Computing. Vienna: R Foundation for Statistical Computing.

Sahai, H., and Ageel, M. I. (2000). The Analysis of Variance: Fixed, Random and Mixed Models. Chicago, IL: Birkhäuser. doi: 10.1007/978-1-4612-1344-4

Sargent, J. R., Bell, J. G., Bell, M. V., Henderson, R. J., and Tocher, D. R. (1995). Requirementcriteria for essential fatty acids. J. Appl. Ichthyol. 11, 183-198. doi: 10.1111/j.1439-0426.1995.tb00018.x

Sargent, J. R., Tocher, D. G., and Bell, J. G. (2002). “The lipids. Chapter 4,” in Fish Nutrition, 3rd Edn., eds J. E. Halver, R. W. Hardy (San Diego, CA: Elsevier Science), 182-246.

Segade, Á., Robaina, L., Otero-Ferrer, F., García Romero, J., and Molina Domínguez, L. (2014). Effects of the diet on seahorse (Hippocampus hippocampus) growth, body colour and biochemical composition. Aquaculture Nutrition 21, 807-813. doi: 10.1111/anu.12202

Sheng, J., Lin, Q., Chen, Q., Shen, L., and Lu, J. (2007). Effect of starvation on the initiation of feeding, growth and survival rate of juvenile seahorses,
Hippocampus trimaculatus Leach and Hippocampus kuda Bleeker. Aquaculture 271, 469-478. doi: 10.1016/j.aquaculture.2006.05.061

Shields, R. J., Bell, J. G., Luizi, F. S., Gara, B., Bromage, N. R., and Sargent, J. R. (1999). Natural copepods are superior to enriched Artemia nauplii as feed for halibut larvae (Hippoglossus hippoglossus) in terms of survival, pigmentation and retinal morphology: relation to dietary essential fatty acids. J. Nutr. 129, 1186-1194.

Sorgeloos, P., Dhert, P., and Candreva, P. (2001). Use of the brine shrimp, Artemia spp., in marine fish larviculture. Aquaculture 200, 147-159. doi: 10.1016/S00448486(01)00698-6

Therneau, T. (2014). A Package for Survival Analysis in S. R package version 2.37-7. Available online at: http://CRAN.R-project.org/package=survival

Tipton, K., and Bell, S. S. (1988). Foraging patterns of two syngnathid fishes: importance of harpacticoid copepods. Mar. Ecol. Prog. Ser. 47, 31-43. doi: 10.3354/meps047031

Treece, G. D. (2000). "Artemia Production for Marine Larval Fish Culture," in Southern Regional Aquaculture Center, Publication No. 702 (Southern Regional Aquaculture Center Stoneville). Available online at http://biomasaxy. com/Acuacultura/Docs/Plancton/Cultivo\%20de\%20Artemia.pdf （Accessed: 26.08.2013).

Ullrey, D. E. (1995). "Fish. Chapter 27," in Basic Animal Nutrition and Feeding, 4th $E d n$, eds W. G. Pond, D. C. Church, and K. R. Pond (Hoboken, NJ; New York, NY: Wiley), 547-568.

Van der Meeren, T., Olsen, R. E., Hamre, K., and Fyhn, H. J. (2008). Biochemical composition of copepods for evaluation of feed quality in production of juvenile marine fish. Aquaculture 274, 375-397. doi: 10.1016/j.aquaculture.2007.11.041

Vincent, A. C. J. (1996). The International Trade in Seahorses. TRAFFIC International. Available online at: http://www.traffic.org/species-reports/ traffic_species_fish33.pdf (Accessed: 26.08.2013).

Vincent, A. C. J., Foster, S. J., and Koldewey, H. J. (2011). Conservation and management of seahorses and other Syngnathidae. J. Fish Biol. 78, 1681-1724. doi: 10.1111/j.1095-8649.2011.03003.x

Wabnitz, C., Taylor, M., Green, E., and Razak, T. (2003). "From ocean to aquarium," in The Global Trade in Marine Ornamental Species (Cambridge: UNEP-WCMC). Available online at: http://www.unep.org/pdf/from_ocean_ to_aquarium_report.pdf (Accessed: 26.08.2013).

Watanabe, T. (1993). Importance of docosahexaenoic acid in marine larval fish. J. World Aquac. Soc. 24, 152-161. doi: 10.1111/j.1749-7345.1993.tb00004.x

Willadino, L., Souza-Santos, L. P., Mélo, R. C. S., Brito, A. P., Barros, N. C. S., Araújo-Castro, C. M. V., et al. (2012). Ingestion rate, survival and growth of newly released seahorse Hippocampus reidi fed exclusively on cultured live food items. Aquaculture 360-361, 10-16. doi: 10.1016/j.aquaculture.2012. 06.025

Wittenrich, M. L. (2007). The Complete Illustrated Breeder's Guide to Marine Aquarium Fishes. Neptune City, NJ; New York, NY: T.F.H. Publications and MICROCOSM.

Wong, J. M., and Benzie, J. A. H. (2003). The effects of temperature, Artemia enrichment, stocking density and light on the growth of juvenile seahorses, Hippocampus whitei (Bleeker, 1855), from Australia. Aquaculture 228, 107-121. doi: 10.1016/S0044-8486(03)00320-X

Woods, C. (2000). Preliminary observations on breeding and rearing the seahorse Hippocampus abdominalis (Teleostei: Syngnathidae) in captivity. N.Z. J. Mar. Freshw. Res. 34, 475-485. doi: 10.1080/00288330.2000.9516950

Woods, C. (2003). Effects of varying Artemia enrichment on growth and survival of juvenile seahorses, Hippocampus abdominalis. Aquaculture 220, 537-548. doi: 10.1016/S0044-8486(02)00639-7

Conflict of Interest Statement: The authors declare that the research was conducted in the absence of any commercial or financial relationships that could be construed as a potential conflict of interest.

Copyright $\odot 2016$ Schubert, Vogt, Eder, Hauffe and Wilke. This is an open-access article distributed under the terms of the Creative Commons Attribution License (CC $B Y$ ). The use, distribution or reproduction in other forums is permitted, provided the original author(s) or licensor are credited and that the original publication in this journal is cited, in accordance with accepted academic practice. No use, distribution or reproduction is permitted which does not comply with these terms. 